\title{
Clinical and Therapeutic Studies of Acquired Thrombotic Thrombocytopenic Purpura in China
}

\author{
Zhaoyue Wang ${ }^{1}$, Ling Sun ${ }^{1}$, Ziqiang Yu ${ }^{1}$, Jian Su${ }^{1}$, Jing Wang ${ }^{2}$, Haifei Chen ${ }^{2}$ and Changgeng Ruan ${ }^{1^{*}}$ \\ ${ }^{1}$ Innovation Centre of Hematology and the Key Laboratory of Thrombosis and Hemostasis of the Ministry of Health, Jiangsu Institute of Hematology, The First Affiliated \\ Hospital of Soochow University, Suzhou, Jiangsu Province, China \\ ${ }^{2}$ Department of Hematology, PLA 100th Hospital, Suzhou, Jiangsu Province, China
}

"Corresponding author: Changgeng Ruan, Innovation Centre of Haematology and the Key Laboratory of Thrombosis and Haemostasis of the Ministry of Health, Jiangsu Institute of Haematology, The First Affiliated Hospital of Soochow University, 188 Shizi Street, Suzhou 215006, Jiangsu Province, China, Tel: 86-512-65113556; Fax: 86-512-65113556; E-mail: changgengruan@hotmail.com

Received date: June 01, 2015, Accepted date: June 22, 2015, Publication date: June 26, 2015

Copyright: (c) 2015 Wang Z, et al. This is an open-access article distributed under the terms of the Creative Commons Attribution License, which permits unrestricted use, distribution, and reproduction in any medium, provided the original author and source are credited.

\begin{abstract}
Thrombotic thrombocytopenic purpura (TTP) is a rare and severe disorder mediated by autoantibodies against ADAMTS13. In this report, we study the clinical features, laboratory aberration, and treatment effect of 55 patients with acquired TTP in China. The classic pentad occurred in only 33\% of TTP patients. Severe ADAMTS13 deficiency was detected in $85 \%$ of patients. Advanced age and hyperbilirubinemia might be risk factors for poor prognosis. Early and sufficient plasma exchange is the most important approach. The addition of rituximab to plasma exchange and corticosteroids appears to be effective in inducing and sustaining long-term remission in TTP, and is suitable to be administrated during the first episode. However, more optimal therapeutic regimen warrants further investigation to treat refractory cases and to reduce relapse rate.
\end{abstract}

Keywords: Thrombotic thrombocytopenic purpura; ADAMTS13; Clinical characteristics; Plasma exchange; Rituximab

\section{Introduction}

Thrombotic thrombocytopenic purpura (TTP) is a rare severe disorder, the incidence of which is estimated to be 3.7 per million [1]. It is characterized by microangiopathic hemolytic anemia, thrombocytopenia and various clinical symptoms, especially in association with neurological and renal damages $[2,3]$. TTP is mainly associated with a severe deficiency of ADAMTS13 activity, while there are some patients who fulfil the diagnostic criteria for TTP do not have severe ADAMTS13 deficiency. TTP is almost always acquired, congenital TTP caused by a mutation of the ADAMTS13 gene is quite rare. In the acquired TTP, a small proportion of patients are associated with other diseases, while the remaining patients are categorized as idiopathic.

Rapid and widespread advances have been made in clinical and fundamental researches on TTP since identifying a deficiency of ADAMTS13 activity related to TTP [4,5], and confirming the efficacy of plasma exchange (PE), which increases the survival rate of patients with TTP from $10 \%$ to about $80 \%[6,7]$.

Corticosteroids and other immunosuppressive agents have also been widely used in the treatment of TTP, but there is scanty unanimous evidence documenting their efficacy. In China, most published literature is still only of case reports or small retrospective studies, mainly because measurements of ADAMTS13 activity have not yet become popular. In this report, we comprehensively analyzed the clinical characteristics, treatment strategies and outcomes of 55 patients with TTP.

\section{Patients and Methods}

\section{Patients}

Fifty-five patients (36 female and 19 male) with a median age of 41 years (range: 13-90) diagnosed between January 2007 and November 2012 were included in this retrospective study. The diagnosis of TTP was established according to Guidelines on the diagnosis and management of the thrombotic microangiopathic haemolytic anaemias by British Society for Haematology [3]. Informed consent to perform the studies was obtained from each patient, and ethical approval was obtained from the ethics committee of our institute according to the Declaration of Helsinki.

The clinical and laboratory features of these patients were variable. Bleeding due to thrombocytopenia included mucocutaneous purpura, epistaxis, gum bleeding, hematuria, melena, and menorrhagia. The manifestations of hemolytic anemia included icterus, hyperbilirubinemia and raised lactate dehydrogenase (LDH) level. Neurological manifestations were headache, altered mental state, confusion, paresthesia, epilepsy, stroke and coma. Renal impairment was revealed as proteinuria, hematuria, raised levels of serum creatinine and urea nitrogen, and renal failure.

\section{Laboratory examinations}

Laboratory indexes were regularly observed such as platelet count, $\mathrm{LDH}$ level, and striking red cell fragmentation. Platelet recovery time was defined as time from treatment with plasma exchange and corticosteroids to a normal platelet count $\left(>100 \times 10^{9} / \mathrm{L}\right)$ for at least 3 consecutive days. LDH recovery time was defined as time from effective treatment to a normal LDH level (100-225 U/L) for at least 3 consecutive days. The recovery time of red cell fragmentation was defined when fragmented red cells could not be detected in blood film. 
Citation: Wang Z, Sun L, Yu Z, Su J, Wang J, et al. (2015) Clinical and Therapeutic Studies of Acquired Thrombotic Thrombocytopenic Purpura in China. J Blood Disord Transfus 6: 288. doi:10.4172/2155-9864.1000288

Page 2 of 4

\section{Measurement of plasma ADAMTS13 activity}

Peripheral blood samples of both TTP patients before the initiation of first plasma exchange and healthy donors were collected with onetenth volume of $3.2 \%$ trisodium citrate. The samples were centrifuged at $\mathrm{g}$ for $10 \mathrm{~min}$ to prepare platelet-poor plasma, which was then kept at -30 until analysis. Plasma ADAMTS13 activity was measured by residual-collagen binding assay according to the method of Rick [8]. Less than $5 \%$ of ADAMTS13 activity was considered as severe deficiency, and the presence of ADAMTS13 inhibitor was assessed in all samples with its severe deficiency.

\section{B lymphocytes detection using flow cytometry}

The flow cytometry measurement of B lymphocytes was performed using EDTA-anticoagulant peripheral blood of the patients treated with retuximab. The white blood cells were adjusted to $(0.5-1) \times 10^{9}$ cells $/ \mathrm{ml}$. Samples of $100 \mu \mathrm{L}$ were incubated for $15 \mathrm{~min}$ in the dark with saturating amounts of fluorescent MoAbs anti-CD3, CD19, CD20 and CD45 (Immunotech, France), and then washed with hemolytic agent to lysis red blood cells. Cells were analyzed by a EpicsXL flow cytometry (Beckman Coulter, USA). Total events of 10000 were gated based on forward (FSC) and side-scatter (SSC) characteristics and dot plots for B lymphocytes were gated.

\section{Response criteria}

Response criteria to treatment was defined according to that described by Zhan [9] and Gurkan [10]: 1. complete response: a platelet count $>100 \times 10^{9} / \mathrm{L}$ and no new clinical events; 2 . partial response: a platelet count $\geq 50 \times 10^{9} / \mathrm{L}$ or more than doubled the baseline count, and no new clinical events; 3 . no response: a platelet count $<20 \times 10^{9} / \mathrm{L}$ or less than doubled the baseline count, and deterioration of the patient's clinical status; and 4. relapse: recurrence of clinical manifestations and laboratory abnormalities after a complete remission for 30 days or longer.

\section{Statistical analysis}

Data were expressed as the median and the range. Comparisons between response group and death group were made using Student's ttest. A two-tailed $\mathrm{p}$-value $<0.05$ was considered statistically significant. Relevant statistical analyses were performed by GraphPad Prism 5 software.

\section{Results}

\section{Clinical features}

Among the 55 patients in our cohort with TTP, 10 (18\%) had autoimmune disorders, $6(11 \%)$ had infection, and pregnancy was associated with 4 patients (7\%). The remaining 35 patients $(64 \%)$ had no obvious underlying causes and were considered to have idiopathic TTP.

Symptoms of the patients with TTP came on abruptly and progressed quickly. The clinical features at the onset included fever, fatigue, thrombocytopenia, hemolytic anemia, and neurological abnormalities, which could occur either simultaneously or subsequently, and exacerbate until initiation of plasma exchange. Clinical variants appeared upon admission. All patients had thrombocytopenia with $74 \%$ showing bleeding phenomena, 51(91) of patients had microangiopathic hemolytic anemia, 45 (82\%) had neurologic abnormalities, 37 (72\%) had fever, and 36 (65\%) had renal abnormalities. Among our cohort, 18 patients (33\%) exhibited the typical pentad of clinical features (Table 1).

\begin{tabular}{|l|l|}
\hline Clinical manifestations & $\mathbf{n}(\%)$ \\
\hline Bleeding & $41(74 \%)$ \\
\hline skin ecchymosis & $24(47 \%)$ \\
\hline hematuria & $5(8 \%)$ \\
\hline epistaxis & $5(8 \%)$ \\
\hline Gl bleeding & $3(6 \%)$ \\
\hline Menorrhagia & $2(4 \%)$ \\
\hline Gum bleeding & $1(2 \%)$ \\
\hline Microangiopathic hemolytic anemia & $51(91 \%)$ \\
\hline Neurological manifestations & $45(82 \%)$ \\
\hline Confusion, coma & $28(51 \%)$ \\
\hline Altered mental state & $10(18 \%)$ \\
\hline Headache, vomiting & $6(10 \%)$ \\
\hline Delirium, convulsion & $5(8 \%)$ \\
\hline Paresthesia & $5(8 \%)$ \\
\hline Delusion & $1(2 \%)$ \\
\hline Fever & $37(67 \%)$ \\
\hline Renal impairment & $36(65 \%)$ \\
\hline Gl: Gastrointestinal & \\
\hline
\end{tabular}

Table 1: Clinical manifestations of TTP patients.

\section{Laboratory findings}

At presentation, the median platelet count was $11 \times 10^{9} / \mathrm{L}$ (range, 5 $\times 10^{9} / \mathrm{L}-72 \times 10^{9} / \mathrm{L}$ ), the median hemoglobin level was $71 \mathrm{~g} / \mathrm{L}$ (range, $50 \mathrm{~g} / \mathrm{L}-156 \mathrm{~g} / \mathrm{L}$ ) with $77 \%$ of patients having moderate to severe anemia. Red cell fragmentation was detected in $86 \%$ of patients with median percentage of $5.0 \%$ (range, 1-15\%).

The median total bilirubin level of plasma was elevated to 49.9 $\mu \mathrm{mol} / \mathrm{L}$ (range, $7.7 \mu \mathrm{mol} / \mathrm{L}$ to $153.1 \mu \mathrm{mol} / \mathrm{L}$ ), and the median $\mathrm{LDH}$ concentration was $1166.4 \mathrm{U} / \mathrm{L}$ (range, $109 \mathrm{U} / \mathrm{L}-2953 \mathrm{U} / \mathrm{L}$ ), with 29 patients (53\%) having $\mathrm{LDH}>1000 \mathrm{U} / \mathrm{L}$. median serum creatinine concentration of 40 patients with renal impairement or renal failure was $167.4 \mu \mathrm{mol} / \mathrm{L}$ (range, $34.8 \mu \mathrm{mol} / \mathrm{L}-735.0 \mu \mathrm{mol} / \mathrm{L}$ ), and median urea nitrogen concentration was $13.1 \mathrm{mmol} / \mathrm{L}$ (range, $4.1 \mathrm{mmol} / \mathrm{L}-49.1$ $\mathrm{mmol} / \mathrm{L}$ ). The results of coagulation tests were generally normal, only three patients showed mild prolongation of activated partial thromboplastin time or thrombin time.

Forty-one patients had ADAMTSl3 activity measured on their admission. In 35 (85\%) patients, ADAMTS13 activity was confirmed to be $<5 \%$, of whom 25 (61\%) were antibody-positive; the remaining 6 patients (15\%) showed normal ADAMTS13 activity including one who had received PE. 


\section{Treatment}

Three of 55 patients died during the first acute episode before sufficient PE therapy. One patient died who was treated only with corticosteroid. Other 51 received plasma exchange with or without corticosteroid combined with such immunosuppressive agents as vincristine, cyclophosphamide, and cyclosporine. Among them, 9 patients were also treated with additional rituximab $\left(375 \mathrm{mg} / \mathrm{m}^{2}\right.$, once weekly for 4 weeks) (Table 2).

In most of 40 survivors (73\%), platelet count was increased after second or third daily $\mathrm{PE}$ and then reached normal range $\left(>100 \times 10^{9} / \mathrm{L}\right)$, followed by decrease of the high LDH level. In our cohort, 15 patients (27\%) died during the disease course. The death rate in patients receiving only $\mathrm{PE}$ was $55 \%$, that in patients treated with plasma exchange in combination with corticosteroids and immunosuppressants was $18 \%$. Nine patients treated with additional rituximab are all surviving (Table 2). In analyzing prognostic risk factors, we found that the mean age of the death group was older than the remission group, and total bilirubin level was higher in the death group, while other characteristics on admission in the two groups were similar such as body temperature, platelet count, white cell count, hemoglobin level, creatinine and LDH (Table 3).

\begin{tabular}{|l|l|l|l|}
\hline Therapeutic regimens & $\mathbf{n}$ & remission (\%) & death (\%) \\
\hline No treatment & 1 & 0 & $1(100 \%)$ \\
\hline Steroids & 3 & 0 & $3(100 \%)$ \\
\hline PE & 9 & $4(45 \%)$ & $5(55 \%)$ \\
\hline PE+steroids+IP & 33 & $27(82 \%)$ & $6(18 \%)$ \\
\hline PE+steroids+IP+rituximab & 9 & $9(100 \%)$ & $0(0 \%)$ \\
\hline $\begin{array}{l}\text { PE: Plasma Exchange; IP: Immunosuppressive } \\
\text { cyclophosphamide or cyclosporine) }\end{array}$ & Agents (vincristine, \\
\hline
\end{tabular}

Table 2: Effect of different therapeutic regimens in TTP patients.

\begin{tabular}{|l|l|l|l|}
\hline Clinical and lab index & Remission $(\mathrm{n}=40)$ & Death $(\mathrm{n}=15)$ & P value \\
\hline Age $(\mathrm{y})$ & $37.5 \pm 14.5$ & $50.1 \pm 18.9$ & 0.008 \\
\hline Temperature $\left({ }^{\circ} \mathrm{C}\right)$ & $38.0 \pm 1.0$ & $37.9 \pm 0.8$ & 0.957 \\
\hline WBC $\left(\times 10^{9} / \mathrm{L}\right)$ & $11.2 \pm 6.4$ & $13.3 \pm 9.7$ & 0.485 \\
\hline Hemoglobin $(\mathrm{g} / \mathrm{L})$ & $71.4 \pm 15.4$ & $85.7 \pm 38.2$ & 0.086 \\
\hline Platelet $\left(\times 10^{9} / \mathrm{L}\right)$ & $15.8 \pm 13.9$ & $14.4 \pm 10.32$ & 0.630 \\
\hline Total bilirubin $(\mu \mathrm{mol} / \mathrm{L})$ & $43.3 \pm 23.5$ & $63.7 \pm 37.7$ & 0.036 \\
\hline Creatinine $(\mu \mathrm{mol} / \mathrm{L})$ & $145.8 \pm 124.9$ & $152.8 \pm 131.5$ & 0.999 \\
\hline Urea nitrogen $(\mu$ mol/L & $11.3 \pm 6.2$ & $13.8 \pm 10.9$ & 0.444 \\
\hline LDH $(\mathrm{U} / \mathrm{L})$ & $1091.9 \pm 585.0$ & $1328.9 \pm 659.3$ & 0.098 \\
\hline LDH = lactate dehydrogenase & & \\
\hline
\end{tabular}

Table 3: Influence of clinical and laboratory indexes on prognosis.

\section{Alteration of B lymphocyte percentage during rituximab treatment}

The B lymphocyte percentage in the peripheral blood before treatment was $18.39 \pm 7.15 \%$, which was decreased to $2.19 \pm 5.11 \%$, $0.53 \pm 1.16 \%$ and $0.14 \pm 0.40 \%$ after the first, second and fourth administration of rituximab, respectively ( $\mathrm{P}$ values $<0.001$ ).

\section{Discussion}

The diagnosis of TTP is mainly based on the presence of thrombocytopenia and microangiopathic hemolytic anemia, which are caused by pathogenetic formation of platelet microvascular thrombi [3]. Although the classic pentad of TTP diagnosis has been recognized since it was first described by Moschowitz in 1924, recent data confirmed that most patients present the triad of TTP, including hemolytic anemia, thrombocytopenia and neurologic abnormalities, while renal impairment and fever occur only in small proportion of TTP patients $[2,11]$. In this study, we found that only $35 \%$ of patients had the classical pentad of TTP. All these results emphasize that the "classic pentad" of clinical features is not relevant to current practice.

The pathogenic mechanism of TTP has been associated with ADAMTS13 deficiency [12,13], ADAMTS13 deficiency has a high sensitivity and specificity for TTP diagnosis. However, there have been some patients in almost all reports who had normal ADAMTS13 activity. In our cohort, $17 \%$ patients fulfilled diagnostic criteria for TTP, but did not have severe ADAMTS13 deficiency. Documentation of a severe deficiency of plasma ADAMTS13 activity is important, but not essential for the diagnosis of TTP. Therefore, its diagnosis should be based on the presence of clinical symptoms, laboratory aberrations consistent with MAHA (MicroAngiopathic Hemolytic Anemia), decreased ADAMTS13 activity, and possibly presence of antiADAMTS13 autoantibodies [14].

PE has been the mainstay of treatment of acute TTP and decreased the mortality rates from $90 \%$ to $10-20 \%$. Meanwhile corticosteroids have widely been used as an adjunctive treatment combined with plasma exchange. On the other hand, other immunosuppressive agents are often used in the treatment of refractory or recurrent TTP [3]. In this study, the mortality rate in patients who were solely treated with plasma exchange was as high as $55 \%$, certainly because PE in China is still logistically difficult that leads limited plasma supplement and an unavoidable delay. However, we found that, in this case, PE in combination with corticosteroid and immunosuppressive agents could significantly increase the remission rate to $81 \%$. Other 9 patients treated with rituximab in addition to $\mathrm{PE}$, corticosteroid and immunosuppressive agents were all in remission, and no relapses occurred during the follow-up period of 5-61 months. Rituximab binds to the CD20 antigen on B lymphocytes and results in $\mathrm{F} c \gamma$ mediated B-cell lysis. In this study, rituximab significantly eradicated $B$ lymphocytes which are involved in the production of the ADAMTS13 auto-antibody. Our results suggest that a role for the early administration of immunosuppressants, especially rituximab, would be advocated. Recent data have also demonstrated the benefit of the addition of rituximab to standard therapy in sustaining long-term remission in TTP $[15,16]$.

There have been several studies about prognostic factors of TTP. Patients with higher titers of anti-ADAMTS13 auto-antibodies and severe thrombocytopenia might be at greater risk for complications and death [17-19]. Dervenoulas et al. [20] reported advanced age and severe renal impairment as the only parameters associated with 
treatment failure and poor outcome. In this study, we found that beside advanced age, hyperbilirubilemia is also a prognostic risk factor. This finding needs to be further investigated by randomized prospective clinical trials conducted with larger series of patients.

Although approximately $80 \%$ of patients can survive in the era of plasma exchange, the survival rate has not changed since then, and many patients have refractory TTP. In addition, risk for relapse is as high as $35 \%$ after remission [2]. More effective as well as safer treatment such as recombinant ADAMTS13 [14] and bortezomib [21] would improve the prognosis of TTP.

Our study has some limitations. First, due to its retrospective nature, the follow-up is not adequate to evaluate a long-term outcome such as disease relapse. Second, the method used for measuring ADAMTS13 was residual-collagen binding assay, which is less sensitive. A new and highly sensitive enzyme immunoassay of ADAMTS13 activity has just been established in our institute for further prospective investigation.

\section{Declaration of Conflicting Interest}

The authors declare that there are no conflicts of interest.

\section{Funding}

This work was supported by the National Science Foundation of China (no. 30770917, no. 81070395), the Priority Academic Programme Development of Jiangsu Higher Education Institutions (PAPD), Jiangsu Province's Key Medical Centre (no. ZX201102), and Life and Health Special Funds of the Jiangsu Province's Science and Technology Bureau (no. BL2012005).

\section{References}

1. Török TJ, Holman RC, Chorba TL (1995) Increasing mortality from thrombotic thrombocytopenic purpura in the United States--analysis of national mortality data, 1968-1991. Am J Hematol 50: 84-90.

2. George JN (2010) How I treat patients with thrombotic thrombocytopenic purpura: 2010. Blood 116: 4060-4069.

3. Allford SL, Hunt BJ, Rose P, Machin SJ; Haemostasis and Thrombosis Task Force, British Committee for Standards in Haematology (2003) Guidelines on the diagnosis and management of the thrombotic microangiopathic haemolytic anaemias. Br J Haematol 120: 556-573.

4. Furlan M, Robles R, Galbusera M, Remuzzi G, Kyrle PA, et al. (1998) von Willebrand factor-cleaving protease in thrombotic thrombocytopenic purpura and the hemolytic-uremic syndrome. N Engl J Med 339: 1578-1584.

5. Tsai HM, Lian EC (1998) Antibodies to von Willebrand factor-cleaving protease in acute thrombotic thrombocytopenic purpura. N Engl J Med 339: 1585-1594.

6. Rock GA, Shumak KH, Buskard NA, Blanchette VS, Kelton JG, et al. (1991) Comparison of plasma exchange with plasma infusion in the treatment of thrombotic thrombocytopenic purpura. Canadian Apheresis Study Group. N Engl J Med 325: 393-397.

7. Korkmaz S, Keklik M, Sivgin S, Yildirim R, Tombak A, et al. (2013) Therapeutic plasma exchange in patients with thrombotic thrombocytopenic purpura: a retrospective multicenter study. Transfus Apher Sci 48: 353-358.

8. Rick ME, Molls S, Taylor MA, Krizek DM, White GC 2nd, et al. (2002) Clinical use of a rapid collagen binding assay for von Willebrand factor cleaving protease in patients with thrombotic thrombocytopenic purpura. Thromb Haemost 88: 598-604.

9. Zhan H, Streiff MB, King KE, Segal JB (2010) Thrombotic thrombocytopenic purpura at the Johns Hopkins Hospital from 1992 to 2008: clinical outcomes and risk factors for relapse. Transfusion 50: 868-874.

10. Gurkan E, Baslamisli F, Guvenc B, Kilic NB, Unsal C, et al. (2005) Thrombotic thrombocytopenic purpura in southern Turkey: a singlecenter experience of 29 cases. Clin Lab Haematol 27: 121-125.

11. Deng MY, Zhang GS, Zhang Y, Xiao H, Dai CW, et al. (2013) Analysis of clinical and laboratory characteristics in 42 patients with thrombotic thrombocytopenic purpura from a single center in China. Transfus Apher Sci 49: 447-452.

12. Fujikawa K, Suzuki H, McMullen B, Chung D (2001) Purification of human von Willebrand factor-cleaving protease and its identification as a new member of the metalloproteinase family. Blood 98: 1662-1666.

13. Levy GG, Nichols WC, Lian EC, Foroud T, McClintick JN, et al. (2001) Mutations in a member of the ADAMTS gene family cause thrombotic thrombocytopenic purpura. Nature 413: 488-494.

14. Sayani FA1, Abrams CS2 (2015) How I treat refractory thrombotic thrombocytopenic purpura. Blood 125: 3860-3867.

15. Ling HT, Field JJ, Blinder MA (2009) Sustained response with rituximab in patients with thrombotic thrombocytopenic purpura: a report of 13 cases and review of the literature. Am J Hematol 84: 418-421.

16. Blombery P, Scully M (2014) Management of thrombotic thrombocytopenic purpura: current perspectives. J Blood Med 5: 15-23.

17. Kremer Hovinga JA, Vesely SK, Terrell DR, Lämmle B, George JN (2010) Survival and relapse in patients with thrombotic thrombocytopenic purpura. Blood 115: 1500-1511.

18. Zheng XL, Kaufman RM, Goodnough LT, Sadler JE (2004) Effect of plasma exchange on plasma ADAMTS13 metalloprotease activity, inhibitor level, and clinical outcome in patients with idiopathic and nonidiopathic thrombotic thrombocytopenic purpura. Blood 103: 4043-4049.

19. Rock G, Kelton JG, Shumak KH, Buskard NA, Sutton DM, et al. (1998) Laboratory abnormalities in thrombotic thrombocytopenic purpura. Canadian Apheresis Group. Br J Haematol 103: 1031-1036.

20. Dervenoulas J, Tsirigotis P, Bollas G, Pappa V, Xiros N, et al. (2000) Thrombotic thrombocytopenic purpura/hemolytic uremic syndrome (TTP/HUS): treatment outcome, relapses, prognostic factors. A singlecenter experience of 48 cases. Ann Hematol 79: 66-72.

21. van Balen T, Schreuder MF, de Jong H, van de Kar NC (2014) Refractory thrombotic thrombocytopenic purpura in a 16-year-old girl: successful treatment with bortezomib. Eur J Haematol 92: 80-82.
This article was originally published in a special issue, entitled: "Thrombolic \& Thrombocytopenic Purpura", Edited by Han-Mou Tsai, Pennsylvania State University, USA 